\title{
Three Dimensional Analysis of Carving Front Turn of Alpine and Boarder-cross Snowboarder
}

\author{
Jinho Back, Hyomin Kang, Wonil Son, Senghak Jo, Jusung Lee,* and Siddhartha \\ Bikram Panday \\ Department of Leisure Sports, Kangwon National University, \\ Gyodong 1,Samcheok City, Kangwondo Province, Republic of Koré ) \\ taijiljs@nate.com \\ Abstract
}

This paper deals with the three dimensional comparative andyses of the darving front turn of alpine and boarder-cross snowboarders. The research aims to provide winter sports instructors and snowboarders with scientific and quantitative data for evaluation of carving front turn. To achieve the purpose, Alpine and Boarder-cross athete from Korean National Team for 2018 Pyeongchang Winter Olymptes were chosen as subjects. Body segment parameters and 90 coordinate points were selected and referred to calculate the CM and Position of CM in different segments. The turn was divided into 3 events and 2 phases. Three dimensional motion pattern analysis of each segment was performed and was analyzed with kinematical variables such as klapsed time, distance, velocity and angular variables. Comprehensive analysis was condicted and the result of the motion analysis indicated the major features in snowboard carving turn in wo different sporting events. The proposed method and the results can be used as a reference for improvement of the training method and skills in snowboarding

Keywords: Biomechanical Anglysis, Three Dimensional, Snowboard, Carving turn

\section{Introduction}

Snowboarding has been growing as one of the popular winter sports among the youths around the world and has shown an explosive growth in Korea since 90s. Since then we can easily notice their increased numbers in different resorts around Korea. Snowboarding can be mainly categorized into 3 categories according to their riding characteristics i.e. Freestyle, Alpine, Boarder-cross [3]. Freestyle boards are produced with more elastic properties to perform junps, tricks and riding in the half pipe whereas, alpine boards are used for downhill speed racing, rotation, and sharp carving turns. Similar to alpine skiing, alpine snowboarding also requires proper posture in order to decrease the air resistance. Moreover, effective jumping skills are needed in order to avoid losing speed depending on the topography of the slope. Boarder cross snowboarding is a mix between speedy alpine snowboarding event and freestyle whereas freestyle ski event, a group of $4 \sim 6$ snowboarders start simultaneously performing rotations, jumps through the inclined course full of obstacles and race to reach the finish line first. Thus, timing is one of the important parts in these two events, where a technically effective turn can play an important role to link and execute another turn. The technical phase of a snowboard turn starts from the traverse up and un-weighting the pressure

This research project was supported by the 2013 Sports Promotion Fund of Seoul Olympic Sports Promotion Foundation from Ministry of Culture, Sports and Tourism |

'Jusung Lee' 
down through the fall line. Different stances and balancing are used for various centring situation. Steering, rotation, pivoting are used to change the direction of the board; edging and turns can be achieved by combination of inclination and angulations $[2,6]$.

Especially, in carving turn which is also one of the advance techniques in snowboarding; edging is very important for the smooth operation of a perfect carved turn. When attempting a carved turn, a snowboarder performs the turn in dynamic equilibrium for balancing the centripetal force and brings the center of mass inside the turn radius. A snowboarder starts the turn from the neutral position where in the first phase adequate inclination is gained by applying pressure to the edge of the board, then moving on to the second phase where the snowboarder rides by shifting the weight to the edge and performs a smooth clean turn [7]. However, depending on the angle of the slope or speed gained and the terrain condition,angle of inclination and un-weighting for a turn as well as pressure varies. Both/skining and snowboarding requires similar basic turn principles with up-down movements as well as requires tilting to bear the centrifugal force and shows similat pattern of angulation for increasing the edge angle as in cycling. As a result, kinematical analysis is required to investigate the effectiveness and suitability of the movement performed in the turn. Moreover, one turn analysis should be conducted prior to the urns which have up-down movement [7]. Reference [1] previously performed a kinematical analysis on angle of joint and joint torque. Whereas, kinematical analysis of snowboard turn's displacement of center of mass, angle of joint of lower limbs and velocity where analyzed by [6] who stressed the importance of analyzing the turn. In addition, [8] conducted studies on snowboard turn control strategy according to expertise whereas, [5] conducted researches on Dynamic analysis and scientific education effects of snowboard. Even though very few researches have been conducted on kinematical and kinetic analysis nowboarding, research analyzing the characteristics of different winter sporting event or the comparative analysis between two different events hasn't been conducted. Hence, the objective of the research is to analyze the motion pattern and behavior in each segment of carying front turn i.e. the displacement of the snowboarder's center of mass, velocity and the joint angle between snowboarders of two different events. Moreover, the study aims on improying the snowboarders' performance by comparative three -dimensional analysis of different kinematical factors and provide quantitative data to winter sports instructors

\section{Method}

In this paper, three dimensional motion analyses (3D) was conducted using Direct Linear Transformation (DLT) method of which kinematic variables of alpine and boarder-cross snowboarder's carving front turn were analyzed.

\subsection{Subjects}

One alpine snowboarder and one boarder-cross snowboarder from Korean National Team for 2018 Pyeongchang Winter Olympics participated in the experiment. Physical characteristics of the study participants are presented in Table 1.

\section{Table 1. Physical Characteristics of the Participants}

\begin{tabular}{|c|c|c|c|c|}
\hline Subject & Height(cm) & Weight(kg) & Age & Experience(yrs) \\
\hline Alpine & 174 & 64 & 19 & 6 \\
\hline Boarder-cross & 174 & 76 & 19 & 4 \\
\hline
\end{tabular}




\subsection{Experimental Procedures}

For the experiment, 1 alpine and 1 boarder-cross snowboarder from Korean National Team for 2018 Pyeongchang Winter Olympics participated. The physical characteristics of the subjects are presented in Table 1. The test took place in the advanced ski slope at P ski resort, Kangwondo Korea. A three dimensional rectangular 5m (width), 8m (length), and $2 \mathrm{~m}$ (height) calibration frame was constructed and were positioned properly to capture the entire turn from the camera's field of view, their motions were recorded using two video cameras of $1.5 \mathrm{~m}$ (height) which were slotted into the tripods. Test recording were conducted for 30 second to 1 minute. The experimental slope was $866 \mathrm{~m}$ in length at an altitude of $238.7 \mathrm{~m}$ with average pitch of $15.41^{\circ}$ to maximum pitch of $36.0^{\circ}$ in the advanced course. The runs were recorded at $30 \mathrm{frames} / \mathrm{sec}$. The time interval of analysis was processed and rearranged at $1 / 100$ of a second due to the possibility of each frame could be divided into two fields of actual speed of 60 fields/sec. The participants were allowed enough time to warm up and time to do two test run to familiarize with the slope conditions and to ensure actual competition condition. Before filming the turns, the experimental procedures and possible risks were communicated verbally and in writing to all study participants, who then gave their informed written consent. In addition, subjects performed two rans of which best turns were selected for analysis. The distance between each gate was about $20 \mathrm{~m}$.

\subsection{Data Analysis}

All the data obtained were processed and analyzed using Kwon 3D 3.1 [4]. When digitizing the coordinates of the calibration frame, reference point was selected from the bottom of the right side and the back side of the pole. 90 coordinate points were selected for analysis. The direction in which the participant descend passing through the gate was selected as $\mathrm{Y}$ axis. While descending vertical progress direction was selected as $\mathrm{Z}$ axis, Left and right direction was selected as $X$ axis i.e. the vector product from $\mathrm{Z}$ axis to $\mathrm{Y}$ axis. Central body joint coordinates is defined as a rigid body system of 23 coordinates of human body model and 16 body segments connected to it. Body segment parameters [9] were referred to calculate the CM and Position of CM in different segments.

\subsection{Definition of Event and Phases}

The selection of event and phase in the turn transition phase in carving turn of alpine snowboarder are presented in Figure 1.

\subsubsection{Definition of Event: $\quad$ E1: Starting point of Extension (UP) \\ E2: Complete contact point of deck surface to the ground \\ E3: Point of Complete flexion (down)}

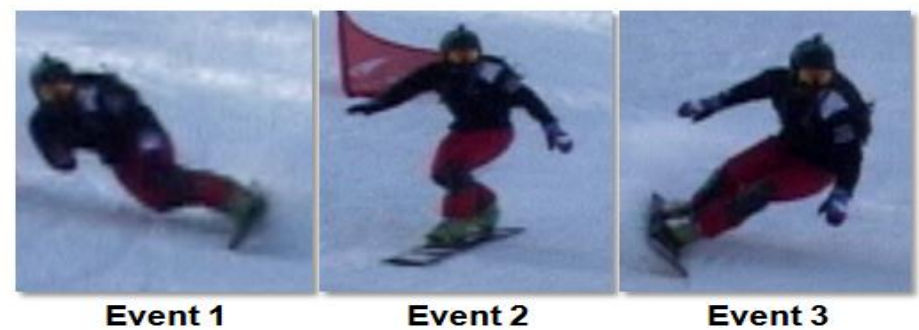

Figure 1. Classification of Movement in Different Events 


\subsubsection{Definition of Phases: P1 (Phase 1): From E1 to E2}

P2 (Phase 2): From E2 to E3

\section{Results and Discussion}

This study was conducted on alpine and boarder-cross snowboarders from Korean National Team for 2018 Pyeongchang Winter Olympics. Video analysis was performed on the snowboarder's front caving turn. Elapsed time, Distance, Velocity and Angular variables were chosen as kinematical variable were analysed. The results from the obtained data are as follows

\subsection{Elapsed Time Variable}

The elapsed time in different phases of carving front turn is presented in Table 2 .

Table 2. Time Elapsed in Different Phases [sec]

\begin{tabular}{|c|c|c|c|}
\hline Subjects & Phase 1 & Phase 2 & Total \\
\hline Alpine & 0.53 & 0.63 & 1.16 \\
\hline Boarder-cross & 0.43 & 0.87 & 1.30 \\
\hline
\end{tabular}

Elapsed time in each phases were dived into two phases and calculated i.e., P1 (from starting of turn to maximum inclination of $\mathrm{CM}$ ) and $\mathrm{P2}$ (Irom maximum inclination of $\mathrm{CM}$ to completion of turn). In P1, alpine snowboarder measured $0.53 \mathrm{sec}$ whereas, boarder-cross snowboarder measured $0.43 \mathrm{sec}$ which is about $0.1 \mathrm{sec}$ faster. However, In P2 alpine snowboarder was $0.24 \mathrm{sec}$ faster than boarder-cross snowboarder. As per result in total elapsed time, alpine snowboarder measured $1.30 \mathrm{sec}$ whereas, boarder-cross measured 1.30 sec. The result obtained is considered as characteristics of alpine snowboarding which is high on edge and has straightness. The alpine snowboarder executed the turn effectively and with higher velocity than the boarde-cross. Whereas, boarder-cross snowboarder performed the turns with more slips which decreased the velocity thus increasing the elapsed time.

\subsection{Distance Variable}

The result of displacement of CM in carving front turn was divided into different events which are presented in Table 3

Table 3. Displacement of CM [m]

\begin{tabular}{|c|c|c|c|c|}
\hline \multirow{3}{*}{ Sulpine } & Axis & Event 1 & Event 2 & Event 3 \\
\cline { 2 - 5 } & $\mathrm{x}$ & 1.85 & 0.27 & -5.8 \\
\cline { 2 - 5 } & $\mathrm{y}$ & -3.09 & 3.45 & 9.50 \\
\cline { 2 - 5 } & $\mathrm{z}$ & 0.63 & 0.43 & 0.82 \\
\hline \multirow{3}{*}{ Boarder-cross } & $\mathrm{x}$ & 2.11 & 1.29 & -6.13 \\
\cline { 2 - 5 } & $\mathrm{y}$ & -3.59 & 1.58 & 9.39 \\
\cline { 2 - 5 } & $\mathrm{z}$ & 0.54 & 0.33 & 0.81 \\
\hline
\end{tabular}

Medial and lateral displacement of CM appeared $1.85 \mathrm{~m}$ in E1, $0.27 \mathrm{~m}$ in E2, $-5.8 \mathrm{~m}$ in E3 of alpine snowboarder whereas, $2.11 \mathrm{~m}$ in E1, $1.29 \mathrm{~m}$ in E2, $-6.13 \mathrm{~m}$ in E3 of boarder-cross snowboarder. Especially, Maximum inclination of CM in E2 appeared lower in alpine than boarder-cross snowboarder with $0.27 \mathrm{~m}$ and $1.29 \mathrm{~m}$ respectively which is considered as an 
effective execution of a turn while approaching closer to the gate. The forward- backward displacement of CM appeared $-3.09 \mathrm{~m}$ in E1, 3.45m in E2, and $9.5 \mathrm{~m}$ in E3 of alpine whereas, $-3.59 \mathrm{~m}$ in E1, $1.58 \mathrm{~m}$ in E2 and $9.39 \mathrm{~m}$ in E3 of boarder-cross snowboarder. Thus, alpine snowboarder measured higher value in maximum inclination of CM in E2 and while completion of the turn in E3 which proves that alpine snowboarder was much further than boarder-cross. The upward-downward displacement of CM was $0.63 \mathrm{~m}$ in E1, $0.43 \mathrm{~m}$ in E2 and $0.82 \mathrm{~m}$ in E3 of alpine snowboarder whereas, $0.54 \mathrm{~m}$ in E1, $0.33 \mathrm{~m}$ in E2 and $0.81 \mathrm{~m}$ in E3 of boarder-cross snowboarder. Hence, decrement in CM and maximum inclination appeared the lowest during the starting phase of the turn (P1) with flexion motion (down) and applying pressure on the board to turn. Whereas, increment in CM appeared by performing an extension motion (up) and releasing in the pressure exerted by the board during the completion of the turn.

\subsection{Velocity Variable}

The results of velocity of $\mathrm{CM}$ and velocity of board in calving front turn of two snowboarders was divided into events which are presented in Table 4 and their patterns in Figure 7-9.

(a)

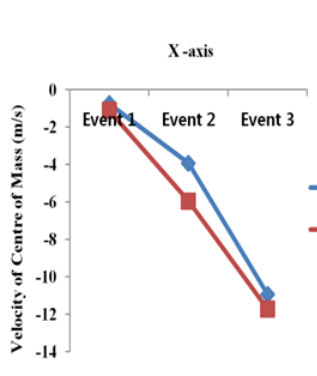

Figure 2. Velocity of Centre of Mlass in meter per second $(m / s)$ of $(a) X$-axis $(b)$ Y-axis (c) Z-axis of Boarder-cross and Alpine Snowboarders in Each Event

The value of medial-laterar velocity of CM appeared highest in boarder-cross than alpine snowboarder which is considered as a phenomenon that appeared in boarder-cross snowboarder to cope with increasing velocity by increasing the angle of inclination as a centripetal force in response to overcome centrifugal force. The forward-backward velocity value appeared higher in alpine than boarder-cross snowboarder. The result obtained is considered as a characteristic of binding setting in alpine snowboarding where on applying pressure on the board during flexion (down) the positioning of the centre of mass moves to front and back. Overall, the upward-downward velocity value appeared higher in alpine than boarder-cross snowboarder which is considered as a characteristic in alpine snowboarding to control the increasing speed through rapid flexion (up) and extension (down) movements to perform an effective turn

\subsection{Angular Variable}

Angle of lower limbs joint, angle of board-hip twist, angle of board- shoulder's twist and upper trunk angle in carving front turn were divided into events their patterns are presented in Figure 3. 
(a)
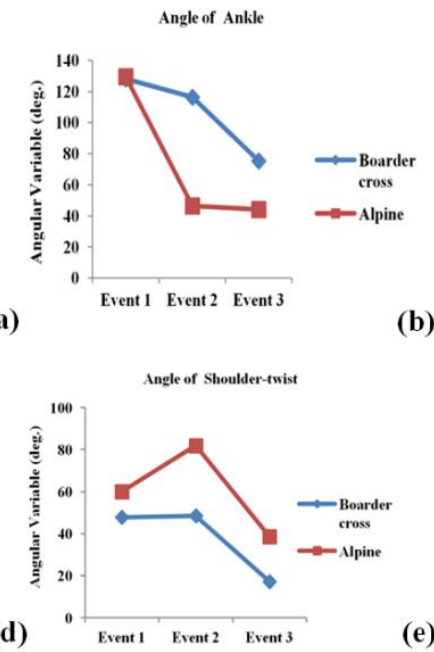

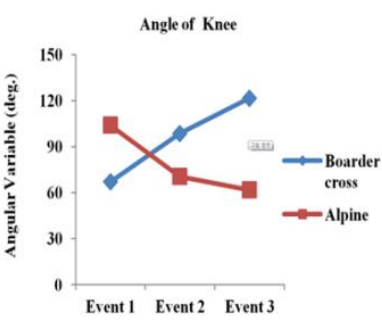

(b)

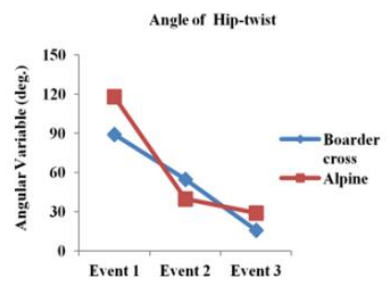

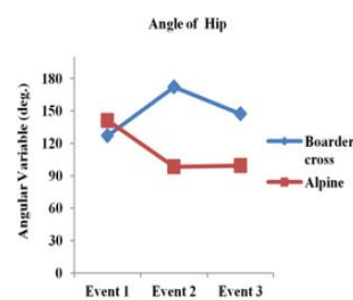

(c)

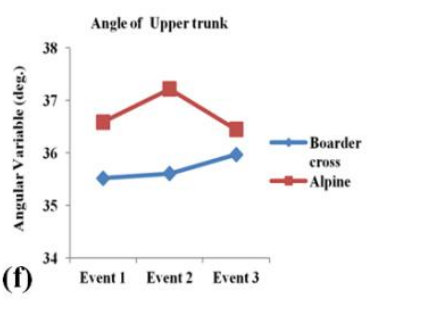

Figure. 3 Angular Variable of Angle of (a) Ankle, (b)Knée, (c) Hip, (d) Shouldertwist, (e)Hip-twist, (f) Upper Trunk in Degree (deg.) of Boarder-cross and Alpine Snowboarders in Each Event

Among the angular variable; angle of ankle, knee and hip-joint appeared lower in alpine than boarder cross as a whole in E2 (the point of maximum inclination of CM). In a study conducted on the experienced and beginner snoyboard instructor by [6] the angle of ankle joint and knee generally appeared low in experienced instructors than in beginner snowboard instructors during a snowboard turn. Thus, Pivoting and rotation was done by alpine snowboarder while skidding to reduce the friction between snow and the board surface. Moreover, through angulation the board moved in the direction of side-cut and showed the propensity of the carving turn. The delgree of twist of the upper and lower body represents the angle of board-shoulder and angle of hip twist which gives natural flow between two consecutive turns. Alpine snowboarder completed the turn with higher value in angle of shoulder-twist whereas, boardet-cross completed the turn with higher value in angle of hiptwist. The differences in the obtained result are considered due to the variation of binding setting and the stance of the two snowboarders. The angle of upper trunk appeared lower in boarder-cross than alpine in all events. The result obtained is considered as a use of inclination oriented turn and lowering of the body for a stable turn

\section{Conclusion}

The sjudy aimed on comparative analysis of the alpine and boarder-cross snowboarder's carving front turn where kinematical variable i.e. distance variable, elapsed time variable, velocity variable, angular variable were analyzed and described to promote better understanding of the carving turn. Moreover, the study aims on providing quantitative data to winter sports instructor to enhance the techniques and movements of the athlete through analyzing the characteristics of the turn in two different sporting events. The conclusion of the comparative study and suggestions are as follows:

In elapsed time variable due to the characteristics of alpine snowboarding which is high on edge and has straightness, the alpine snowboarder executed the turn effectively and with higher velocity than the boarder-cross. Whereas, boarder-cross snowboarder performed the turns with more slips which decreased the velocity thus increasing the elapsed time. In the displacement of centre of mass $(\mathrm{CM})$ in distance variable, maximum inclination of $\mathrm{CM}$ in $\mathrm{E} 2$ 
appeared lower in alpine than boarder-cross snowboarder with $0.27 \mathrm{~m}$ and $1.29 \mathrm{~m}$ respectively which is an effective execution of a turn while approaching closer to the gate. Moreover, alpine snowboarder was much further in position than boarder- cross in each event and proper execution of flexion and extension movement can also be noticed from upward-downward displacement. In the change of velocity of center of mass (CM) in velocity variable, highest value appeared in medal-lateral velocity in boarder-cross than alpine snowboarder. It is considered as a phenomenon that appeared in boarder-cross to cope with increasing velocity by increasing the angle of inclination which is an application of centripetal force in response to overcome the acting centrifugal force. Higher value in forward-backward velocity in alpine snowboarder appeared which is considered as a characteristic of binding setting in alpine snowboarding where on applying pressure on the board during flexion (down) the positioning of the center of mass moves to front and back. As a whole, the upward-downuard velocity value appeared higher in alpine than boarder-cross snowboarder which is considered as a characteristic in alpine snowboarding to control the increasing speed through rapid flexion (up) and extension (down) movements to perform an effective turn. In angular variable; angle of ankle, knee and hip-joint appeared lower in alpime than boarder-cross as a whole in the point of maximum inclination of CM i.e. E2. Alpine showboarder completed the turn with higher value in angle of shoulder-twist whereas, boarder-cross completed the turn with higher value in angle of hip-twist. The angle of upper trunk appeared lower in boarder-cross than alpine in all events. The result obtained is considered as a use of inclination oriented turn and lowering of the body for stable turn. Thus, three dimensional kinematical analysis of carving front turn was performed by alpine and boarder-eross snowboarders. Comprehensive analysis was conducted and results were drawn. Our research pointed out few suggestions to the snowboarders which are as follows:

1) Alpine snowboarder:

- To perform an effective turn, alpne snowboarders should decrease the time of flexion (down).

- In order to control the increasing speed, the snowboarder should lower the stance. Moreover, rotating and pivoting the upper body can increase the stability in a turn.

- In addition, applying strongedging through angulations can reduce slipping thus increasing the effectiveness of a turn.

2) Boarder-cross snowboarder.

- Similarly, in order to overcome centrifugal force in faster speeds, boarder-cross snowboarder should lower the stance.

- In order tô perform an effective turn, the snowboarder should increase the inclination and apply strong edging through angulation which will control the board from getting slipped.

- In addition, through rapid change in angle and speed of hip-twist and proper use of edging willenhance the performance of the snowboarder.

\section{References}

[1] H. Doki, C. Nagail and M. Hokari, "A Study on the Dynamic Analysis of Snowboarding Turn", Transactions of the Japan Society of Mechanical Engineers. C 72.713, pp190-196, Japan, (2006).

[2] Canadian Association of Snowboard Instructors, Canadian Association of Snowboard Instructors guide book, www.casi-acms.com, (2000).

[3] H. P. Platzer, C. Raschner, C. Patterson and S. Lembert, "Comparison of physical characteristics and performance among elite snowboarders", Journal of Strength and Conditioning Research, vol. 23, no. 5, (2009), pp. 1427-1432.

[4] Y.-H. Kwon, "Kwon 3D 3.1 for windows motion analysis package", visol company, Korea, (2003).

[5] S.-H. Youn, "Dynamic analysis and scientific education effect of Snowboard", M.PE. Thesis, Department of Physical Education, Hankuk University of Foreign Studies, Korea, (2007).

[6] H.-D. Jo, "The Kinematic Comparative Analysis of the Snowboard turn"” M.PE. Thesis, Graduate School of Korea National University of Education, Korea, (2011). 
[7] M.-S. Hyun and S.-C. Lee, "Kinematic Analysis of Novice Turn and Carving Turn in Snowboard", Journal of koreawellness, vol. 7, no. 1, Korea, (2012).

[8] B. Y. Lee, "Snowboard turn control strategy according to expertise", M.PE. Thesis, Graduate School of Seoul National University, Korea, (2011).

[9] R. F. Chandler, C. E. Clauser, J. T. McConville,H. M. Reynolds and J. W. Young, "Investigation of Inertial properties of the Human Body", No. AMRL-TR-74-137 Final Rpt. (1975).

\section{Authors}

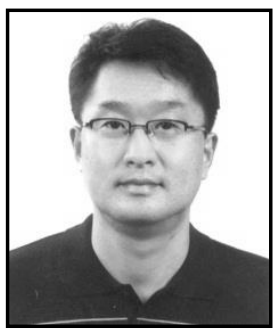

Jin ho Back is a professor at Department of Leisure Sports, Kangwon National University Samcheok Campus. He received ohis Ph.D. degree from Sungkyunkwan University, Korea in 1997. His research interests include Sports Biomechanicsa and Motion Analysis.

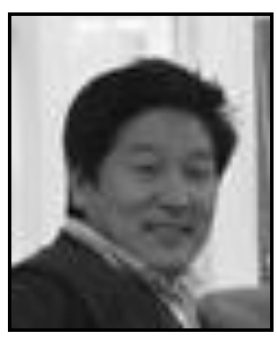

Hyomin Kang is a professor at Department of Leisure Sports, Kangwon National University Samcheok Campus. He received his $\mathrm{PhD}$ Degree from Korea Sports University, Korea. His research interests includes Sports and Culfure, Leisure Sports, Social welfare policy .

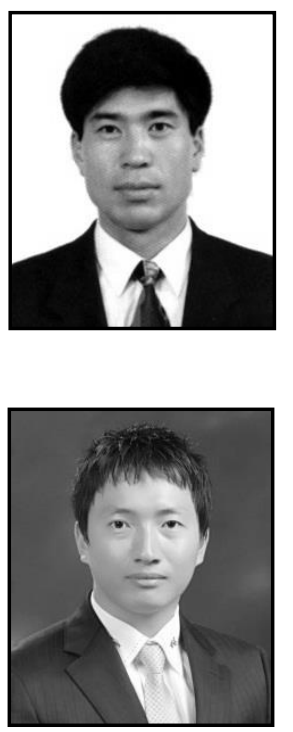

Won Il Son received his Ph.D. degree from Hanyang University, Korea in 1999. Currently, Prof. Son is a faculty member with the Leisure Sports Department, Kangwon National University Samcheok Campus 10 Korea. His research interest includes Physical Evaluation and Measurement.

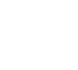

Jusung Lee received his B.Ed and M.Ed in Physical Education from Kangwon National University, Korea in 2009 and 2013 respectively. His field of interest includes Sports Biomechanics, EMG Analysis, Motion Analysis, Motor Control and Disable Sports. 


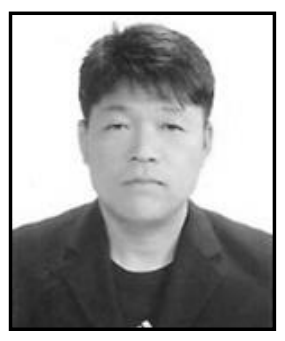

Senghak Jo received his M.Ed in Physical Education from Kangwon National University,Korea. Mr. Jo is a vice president of Kangwondo Football Association for Disabled as well as head coach of Korean Football Association of Mentally Disabled.

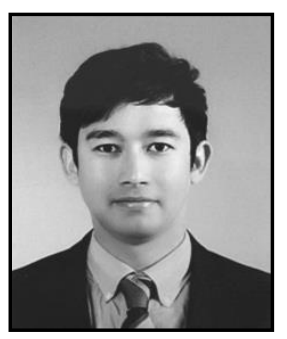

Siddhartha Bikram Panday is currently a graduate student majoring Physical Education at Kangwon National University, Korea. Curpently, $\mathrm{He}$ is a researcher in a project for Development of Measurement and Evaluation in Ski and Snowboard Based on Sports Science which is supported by Ministry of Culture, Sportsand Tourism.

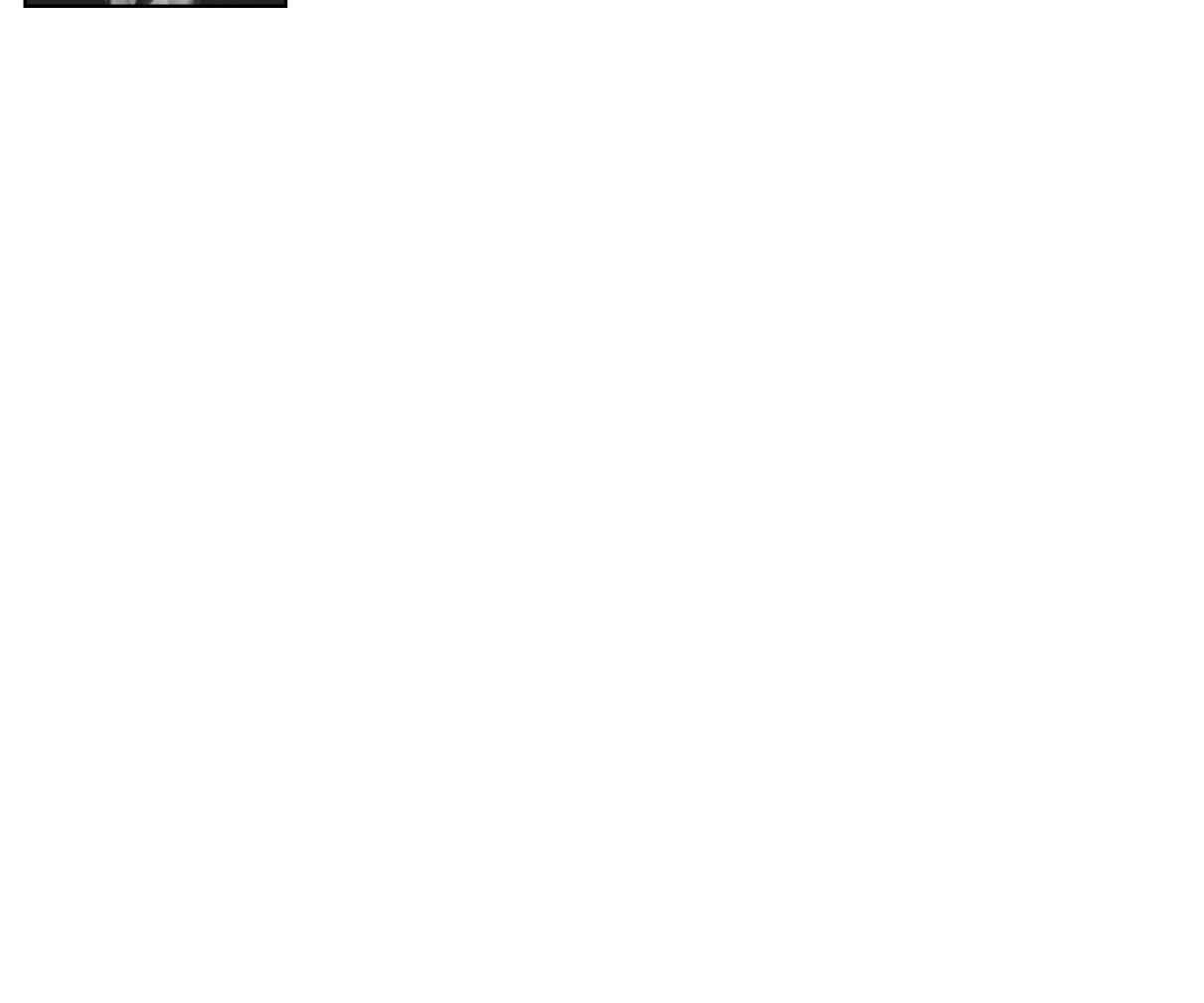


International Journal of Multimedia and Ubiquitous Engineering Vol. 9, No. 10 (2014)

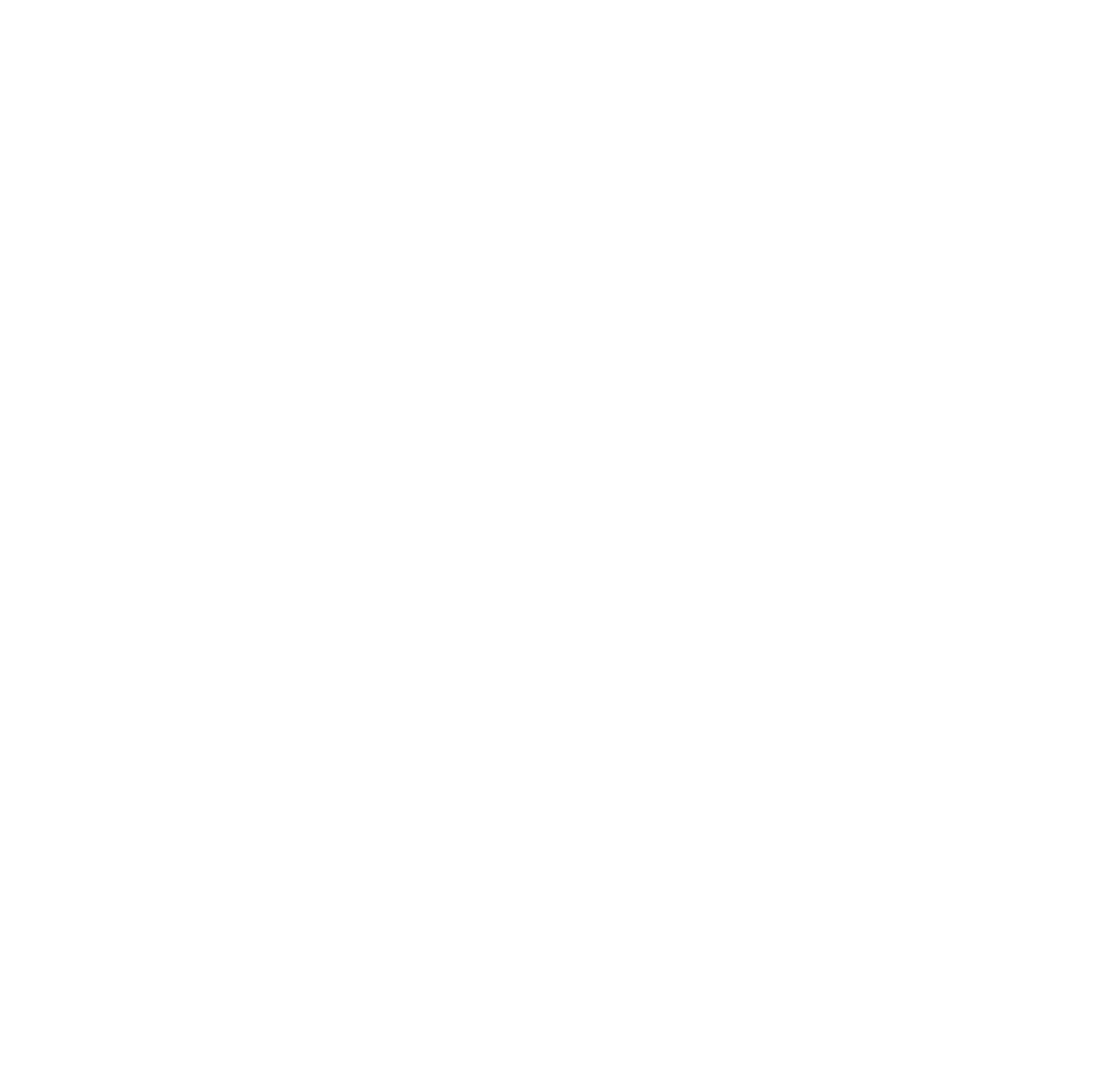

